\title{
Strong exciton-photon coupling in a low-Q all-metal mirror microcavity
}

\author{
P. A. Hobson and W. L. Barnes ${ }^{\text {a) }}$ \\ Thin Film Photonics Group, School of Physics, University of Exeter, Exeter EX4 4QL, United Kingdom
}

D. G. Lidzey, ${ }^{\text {a) }}$ G. A. Gehring, D. M. Whittaker, and M. S. Skolnick

Department of Physics and Astronomy, The University of Sheffield, Sheffield, S3 7RH, United Kingdom

\author{
S. Walker \\ Department of Electrical and Electronic Engineering, The University of Sheffield, Mappin Street, \\ Sheffield S1 3JD, United Kingdom
}

(Received 25 April 2002; accepted 10 September 2002)

\begin{abstract}
We report the experimental observation of strong exciton-photon coupling in a planar microcavity composed of an organic semiconductor positioned between two metallic (silver) mirrors. Via transmission and reflectivity measurements, we observe a very large, room temperature Rabi splitting in excess of $300 \mathrm{meV}$. We show that the Rabi-splitting is enhanced in all-metal microcavities by a factor of more than 2 compared to an organic film positioned between a silver mirror and a dielectric mirror. This enhancement results from the significantly larger optical fields that are confined within all-metal microcavities. (C) 2002 American Institute of Physics.
\end{abstract}

[DOI: $10.1063 / 1.1517714]$

Placing emitters of light such as excitons within microcavities is attractive for device applications and allows the study of new optical phenomena. The effect of the microcavity on the emission of light can be divided into two regimes. In the weak-coupling regime, the spatial and temporal distribution of the emitted radiation can be altered. This regime is employed in applications such as vertical cavity surface emitting lasers and resonant cavity light emitting diodes. ${ }^{1,2}$ In the strong-coupling regime, a mixing between optical and electronic (excitonic) states within the cavity occurs, leading to the appearance of new states termed cavity-polaritons. ${ }^{3}$ This effect is an intensive area of research due in part to the interest in coherent, stimulated effects in such systems that may lead to new optical devices. ${ }^{4}$ The field has recently expanded to include Frenkel excitons supported by organic materials. ${ }^{5-7}$ This has been important in that it has led to the observation of significantly larger, room temperature, strongcoupling effects, and opens the possibility of easily fabricated nonlinear optical devices.

A key phenomenon associated with strong-coupling ${ }^{8}$ is the anticrossing of the exciton and photon mode where, in the absence of a strong interaction, they would have crossed. Until now, investigations into strong-coupling in organic and inorganic materials have been conducted either using two Bragg reflectors (DBR) as microcavity mirrors or one Bragg reflector together with one optically thick metal mirror. In this letter we show that microcavities fabricated using just two metal mirrors can operate in the strong-coupling regime. All-metal cavities are characterized by relatively low $Q$-factors; ${ }^{9}$ however we find that strong-coupling can still be achieved because the effective optical path length in an allmetal cavity is significantly shorter than that in microcavities based on one or more dielectric mirrors, providing a significant enhancement of the optical field within the organic

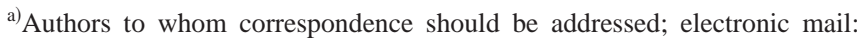
d.g.lidzey@sheffield.ac.uk; w.1.barnes@exeter.ac.uk
}

semiconductor region of the cavity. Because of this enhancement we observe very large Rabi-splittings of over $300 \mathrm{meV}$.

$J$-aggregates of organic dye molecules possess many features that make them particularly suitable to undergo strongcoupling in microcavities. ${ }^{6}$ The narrow, inhomogeneous linewidths (40-50 meV) and very large oscillator strengths of molecular $J$-aggregates allow the observation of strongcoupling at room temperature. To observe the splitting between exciton and photon, both the homogeneous and inhomogeneous linewidth of the cavity photon mode and the excitons must be smaller than the Rabi-splitting. Because of the very large oscillator strength of organic Frenkel excitons (which can be at least 100 times larger than that of a series of III-V quantum wells), we have observed ${ }^{5}$ Rabi-splittings of $160 \mathrm{meV}$ in microcavities having "bare" cavity photon linewidths of around $20 \mathrm{meV}$.

Thin films of the $J$-aggregate forming cyanine dye (2,2' -dimethyl-8-phenyl-5,6,5', $6^{\prime}$,-dibenzothiacarbocyanine chloride, shown as an inset in Fig. 1) were formed by dissolving it into the transparent host polymer polyvinyl alcohol (PVA) in a 40:60 water:methanol mixture, using dye concentrations of order $5 \times 10^{19}$ molecules per $\mathrm{cm}^{3}$. Figure 1 shows the room temperature absorption spectrum obtained from such a PVA matrix film spin cast onto a silica substrate. The microcavities (left insert, Fig. 1) used in our experiment were fabricated by evaporating a thin $(28 \pm 5 \mathrm{~nm})$ silver film onto a silica substrate. The cyanine-dye/PVA mix was then spin cast on top of the metal film, the spin speed being adjusted to produce film thicknesses in the range 175 to $200( \pm 15) \mathrm{nm}$, thus placing the cavity photon mode close to the energy of the exciton mode for normally incident light. Microcavities were completed by evaporating a second thin film of silver $(28 \pm 5 \mathrm{~nm})$ on top of the organic layer, forming a low $Q$ cavity ( $Q \sim 10$ ) of linewidth $\sim 120 \mathrm{meV}$. For comparison, we also fabricated and measured metal-DBR cavities in which the lower mirror (i.e., adjacent to the substrate) was replaced 


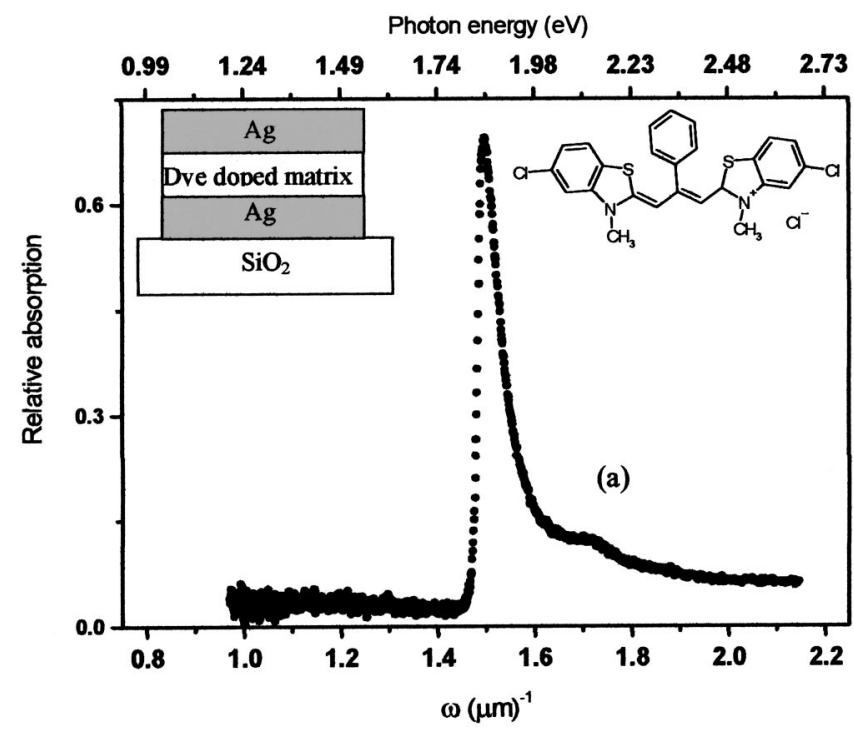

FIG. 1. The absorption spectrum of a thin film of cyanine dye $J$-aggregates dispersed in a PVA matrix. The right inset shows the chemical structure of the cyanine dye studied in this work, a schematic of the planar microcavity structure is shown in the left inset.

by a multilayer dielectric mirror having a peak reflectivity of $98 \%$ at $700 \mathrm{~nm}$.

Microcavities were characterized by measuring their (room temperature) optical transmissivity and reflectivity between angles of incidence of $0^{\circ}$ and $89^{\circ}$. When the frequency and in-plane wave vector of the incident light match that of a microcavity mode, transmission (reflectivity) is resonantly enhanced (reduced). The in-plane wave vector $k_{\|}$is related to the incident angle $\theta$ and wavelength $\lambda$ of the incident light through the relation $k_{\|}=\sin \theta(2 \pi / \lambda)$. Thus, by measuring the transmission as a function of frequency and in-plane wave vector, the dispersion of the cavity modes can be determined (experimental details are given elsewhere ${ }^{10}$ ). Photoluminescence (PL) was also measured as a function of angle following nonresonant excitation by a $594 \mathrm{~nm} \mathrm{HeNe}$ laser, the PL was detected using a CCD spectrometer system.

The data acquired from one sample for TE-polarized transmission are shown in Fig. 2(a) as a gray scale map. High transmission (dark) regions map out the dispersion of the cavity modes. To analyze the optical properties of the cavity we used a standard transfer matrix reflectivity model, into which we input the dielectric functions of the silver mirrors and the $J$-aggregates in the PVA matrix (which we determined from absorption measurements on a control sample). The $J$-aggregate excitons were modelled as oscillators having an absorbance that peaks at $\omega / 2 \pi c$ $=1.49 \mu \mathrm{m}^{-1}(1.84 \mathrm{eV})$. The results of our analysis are shown superimposed on Fig. 2(a). The agreement between the predicted dispersion of the polariton modes and the regions of high cavity transmission are very good, enabling us to identify both the upper and lower polariton branches as shown. Reflectivity measurements of the same structure are shown in Fig. 2(b). The data shown in Figs. 2(a) and 2(b) indicate that both transmission and reflection may be used to study the metal clad structure.

The PL emission from the microcavity as a function of angle is shown in Fig. 3. At $0^{\circ}$, two main features are apparent: a low energy feature centred at $1.69 \mathrm{eV}$, and second (a) Transmission

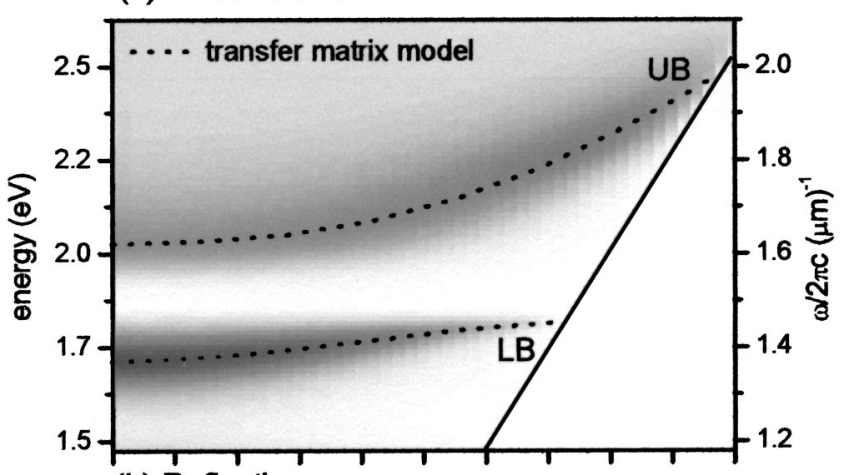

(b) Reflection

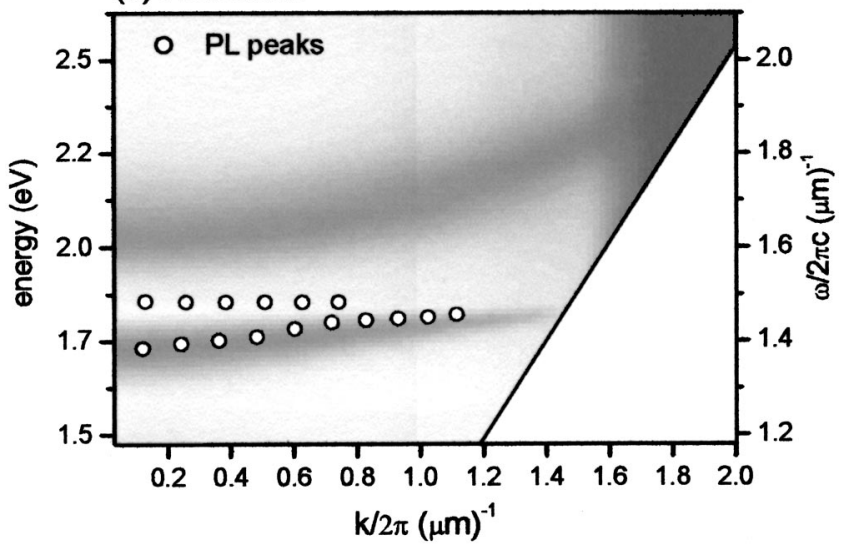

FIG. 2. (a) transmission across the visible region of the spectrum for TE polarized radiation. Dark regions are areas of high transmission. Anticrossing of the cavity modes and the exciton is very clear. The diagonal line on the right of the plot signifies the maximum accessible scan angle $\left(\sim 90^{\circ}\right)$. Also shown in (a) are the results from the transfer matrix model. The dark region beyond $k_{\|}=1.6 \mu \mathrm{m}^{-1}$ is an experimental artifact. (b) reflectivity across the visible region of the spectrum for TE polarized radiation for the same sample as in (a). Dark regions are areas of low reflection. Also shown in are the positions of the peaks obtained from PL data.

sharper feature at $1.85 \mathrm{eV}$. The feature at $1.85 \mathrm{eV}$ corresponds to "direct" emission from the $J$-aggregates within the cavity which do not appear to couple to the optical mode of the structure. At present the origin of this uncoupled emission is not fully understood. However similar emission has been observed in inorganic II-VI semiconductor microcavities, and has been ascribed to emission from localized states. ${ }^{11}$ The lower energy peak at $1.69 \mathrm{eV}$ coincides with the energy of the lower polariton branch. The dispersion of this low energy feature follows the dispersion of the lower polar-

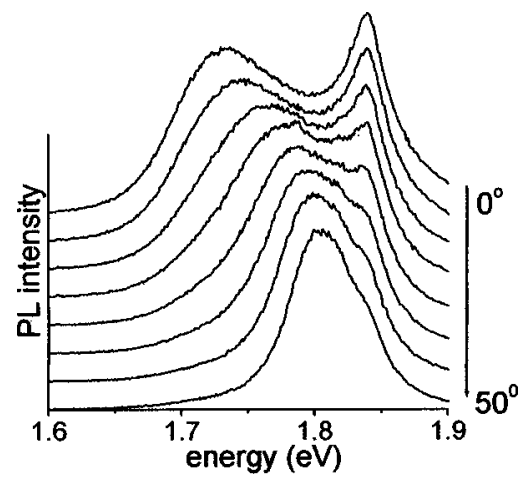

FIG. 3. PL emission spectra recorded from a microcavity containing cyanine $J$-aggregates. The spectra for different angles have been displaced vertically for clarity.

o AIP license or copyright, see http://ojps.aip.org/aplo/aplcr.jsp 


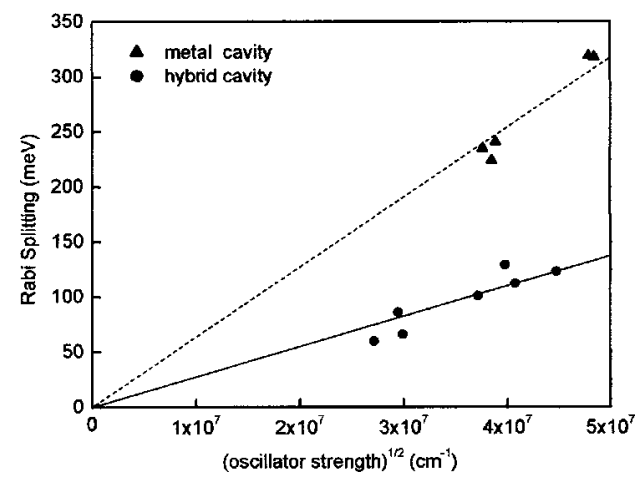

FIG. 4. Measured Rabi-splitting for all-metal and metal-DBR microcavities as a function of the square root of the oscillator strength.

iton branch seen in reflection and transmission. In Fig. 2(b) we indicate the position of the features determined from the PL spectra shown in Fig. 3. As we have observed in other strongly coupled $J$-aggregate microcavities, the photon emission from the lower branch dominates, with emission from the upper branch being much weaker. ${ }^{12}$

From the reflectivity and transmissivity measurements, we determine a Rabi-splitting energy between the polariton branches of $300 \mathrm{meV}$. This value is significantly larger than our previous measurements using the same cyanine dye in a metal-dielectric mirror cavity and is the largest Rabisplitting ever reported in a microcavity. To investigate the enhancement of the Rabi-splitting, we fabricated a series of all-metal cavities and metal-DBR cavities containing $J$-aggregate films having different optical densities, and thus different oscillator strengths. For each cavity, an identical control (noncavity) film of $J$-aggregates in PVA was fabricated. We were able to accurately determine the oscillator strength of each control film (in units of $\mathrm{cm}^{-2}$ ) from a measurement of its absolute optical transmission. In Fig. 4 we plot the square root of the oscillator strength of the organic film within the cavity versus the Rabi-splitting, confirming the expected linear dependence. ${ }^{13}$ It can be seen that both the metal-metal cavity and the metal-dielectric mirror show the anticipated dependence of Rabi-splitting on oscillator strength, however, it is clear that the Rabi-splitting measured in the all-metal cavities is enhanced by $\sim 2.3 \pm 0.3$.

We can explain this increase in splitting on the basis of the reduced optical path length of the metal-metal cavity compared to the metal-dielectric mirror cavity. The optical field within a cavity defined using dielectric mirrors penetrates a significant distance into each dielectric mirror. ${ }^{13,14}$ In the metal-dielectric mirror cavity, we calculate that the optical field penetrates approximately $0.87 \lambda$ into the dielectric mirror thus increasing the effective cavity length $\left(L_{\text {eff }}\right)$. It has been shown ${ }^{15}$ that the Rabi-splitting is proportional to $L_{\text {eff }}^{-1 / 2}$. We calculate that the effective optical path length of the metal-dielectric cavity is approximately 2.7 times longer than the metal-metal cavity, which implies that the Rabisplitting in the metal-metal cavity should be enhanced by a factor of approximately 1.7. A full transfer matrix simulation yields a very similar result. This factor is in reasonable agreement with the measured enhancement in the splitting of approximately 2.3. We therefore explain the enhanced splitting by better confinement of the optical field in the all-metal cavity as the penetration of the optical field into a silver mirror is very small ( $\sim 10 \mathrm{~nm}$ for light of $\lambda=670 \mathrm{~nm})$. This significantly shortens the effective cavity length of the metal-metal cavity, which enhances the optical field that interacts with the $J$-aggregates. The enhancement of the optical field is thus reflected by an increase in the Rabi-splitting.

We have demonstrated that microcavities with all-metal mirrors may be successfully used to explore strong-coupling phenomena, despite the absorption exhibited by the metals and the associated low- $Q$ of such cavities. Indeed, the strength of the splitting is the largest ever measured in a microcavity and is due to both the large oscillator strength of the organic film, and the strong field enhancement in metallic microcavities. The large splitting we obtain is of particular significance for current efforts to achieve polariton lasing in microcavities. Strong-coupling will be maintained in our structures at high temperatures, where the "polariton bottleneck" effect, ${ }^{16}$ which inhibits scattering into polariton states, is much less significant. The benefits of increasing the Rabisplitting are apparent in studies of II-VI microcavities ( $\hbar \omega$ $\sim 30 \mathrm{meV}$ ), where stimulated scattering has been observed for nonresonant excitation. ${ }^{17}$

This work was supported by the UK Engineering and Physical Sciences Research Council and through an Advanced Fellowship award to one of the authors (D.G.L.) The authors wish to thank P. Andrew and D. Bradley for valuable discussions and assistance.

${ }^{1}$ V. Bulovic, V. B. Khalfin, G. Gu, P. E. Burrows, D. Z. Garbuzov, and S. R. Forrest, Phys. Rev. B 58, 3730 (1998).

${ }^{2}$ R. B. Fletcher, D. G. Lidzey, D. D. C. Bradley, M. Bernius, and S. Walker, Appl. Phys. Lett. 77, 1262 (2000).

${ }^{3}$ G. Khitrova, H. M. Gibbs, F. Jahnke, M. Kira, and S. W. Koch, Rev. Mod. Phys. 71, 1591 (1999).

${ }^{4}$ R. M. Stevenson, V. N. Astratov, M. S. Skolnick, D. M. Whittaker, M. Emam-Ismail, A. I. Tartakovskii, P. G. Savvidis, J. J. Baumberg, and J. S. Roberts, Phys. Rev. Lett. 85, 3680 (2000).

${ }^{5}$ D. Lidzey, D. Bradley, M. Skolnick, E. Virgili, S. Walker, and D. Whittaker, Nature (London) 395, 53 (1999).

${ }^{6}$ D. G. Lidzey, D. D. C. Bradley, T. Virgili, A. Armitage, M. S. Skolnick, and S. Walker, Phys. Rev. Lett. 82, 3316 (1999).

${ }^{7}$ D. G. Lidzey, D. D. C. Bradley, A. Armitage, S. Walker, and M. S. Skolnick, Science 288, 1620 (2000).

${ }^{8}$ C. Weisbuch, M. Nishioka, A. Ishikawa, and Y. Arakawa, Phys. Rev. Lett. 69, 3314 (1992).

${ }^{9}$ M. G. Salt, W. C. Tan, and W. L. Barnes, Appl. Phys. Lett. 77, 193 (2000).

${ }^{10}$ M. G. Salt and W. L. Barnes, Opt. Commun. 166, 151 (1999).

${ }^{11}$ M. Muller, J. Bleuse, and R. Andre, Phys. Rev. B 62, 16886 (2000).

${ }^{12}$ D. G. Lidzey, A. M. Fox, M. D. Rahn, M. S. Skolnick, and V. Agranovich, Phys. Rev. B 65, 195312 (2002).

${ }^{13}$ M. S. Skolnick, T. A. Fisher, and D. M. Whittaker, Semicond. Sci. Technol. 13, 645 (1998).

${ }^{14}$ V. Savona, L. C. Andreani, P. Schwendimann, and A. Quattropani, Solid State Commun. 93, 733 (1995).

${ }^{15}$ G. Panzarini, L. C. Andreani, A. Armitage, D. Baxter, M. S. Skolnick, V. N. Astratov, J. S. Roberts, A. V. Kavokin, M. R. Vladimirova, and M. A. Kaliteevski, Phys. Rev. B 59, 5082 (1999).

${ }^{16}$ A. I. Tartakovskii, M. Emam-Ismail, R. M. Stevenson, M. S. Skolnick, V. N. Astratov, D. M. Whittaker, J. J. Baumberg, and J. S. Roberts, Phys. Rev. B 62, R2283 (2000).

${ }^{17}$ A. Alexandrou, G. Bianchi, E. Peronne, B. Halle, F. Boeuf, R. Andre, R. Romestain, and L. S. Dang, Phys. Rev. B 64, 233318 (2001). 